\title{
Defining Success After Surgery for Pelvic Organ Prolapse
}

\author{
Matthew D. Barber, MD, MHS, Linda Brubaker, MD, MS, Ingrid Nygaard, MD, Thomas L. \\ Wheeler II, MD, MSPH, Joeseph Schaffer, MD, Zhen Chen, MS, and Cathie Spino, DSc for the \\ Pelvic Floor Disorders Network \\ Obstetrics, Gynecology, and Women's Health Institute, Cleveland Clinic, Cleveland, Ohio; \\ Departments of Obstetrics \& Gynecology and Urology, Loyola University Chicago, Illinois; \\ Department of Obstetrics and Gynecology, University of Utah, Salt Lake City, Utah; Department of \\ Obstetrics and Gynecology, University of Alabama-Birmingham, Birmingham, Alabama; \\ Department of Obstetrics and Gynecology, University of Texas-Southwestern, Dallas, Texas; Data \\ Coordinating Center, University of Michigan, Ann Arbor, Michigan
}

\begin{abstract}
OBJECTIVES-To describe pelvic organ prolapse surgical success rates using a variety of definitions with differing requirements for anatomic, symptomatic, or re-treatment outcomes.

METHODS—Eighteen different surgical success definitions were evaluated in participants who underwent abdominal sacrocolpopexy within the Colpopexy and Urinary Reduction Efforts trial. The participants' assessments of overall improvement and rating of treatment success were compared between surgical success and failure for each of the definitions studied. The Wilcoxon rank sum test was used to identify significant differences in outcomes between success and failure.
\end{abstract}

RESULTS-Treatment success varied widely depending on definition used (19.2-97.2\%). Approximately $71 \%$ of the participants considered their surgery "very successful," and $85.2 \%$ considered themselves "much better" than before surgery. Definitions of success requiring all anatomic support to be proximal to the hymen had the lowest treatment success (19.2-57.6\%). Approximately 94\% achieved surgical success when it was defined as the absence of prolapse beyond the hymen. Subjective cure (absence of bulge symptoms) occurred in $92.1 \%$ while absence of retreatment occurred in $97.2 \%$ of participants. Subjective cure was associated with significant improvements in the patient's assessment of both treatment success and overall improvement, more so than any other definition considered $(P<.001$ and $<.001$, respectively). Similarly, the greatest difference in symptom burden and health-related quality of life as measured by the Pelvic Organ Prolapse Distress Inventory and Pelvic Organ Prolapse Impact Questionnaire scores between treatment successes and failures was noted when success was defined as subjective cure $(P<.001)$.

CONCLUSION-The definition of success substantially affects treatment success rates after pelvic organ pro-lapse surgery. The absence of vaginal bulge symptoms postoperatively has a significant

Corresponding author: Matthew D. Barber, MD, MHS, Cleveland Clinic, 9500 Euclid Avenue, Desk A81, Cleveland, OH 44195; barberm2@ccf.org.

Presented at the 2009 Annual Meeting of the Society for Gynecologic Surgeons in New Orleans, Louisiana, March 30 to April 1, 2009, and at the 2009 Annual Meeting of the American Urological Association in Chicago, Illinois, April 25-30, 2009. To be presented the 2009 International Continence Society Annual Meeting in San Francisco, California, September 29 to October 3, 2009.

Financial Disclosure

Dr. Brubaker is a consultant and study investigator for Pfizer (New York, NY). Dr. Schaffer has served on the speaker's bureau and national advisory board for Astellas (Toyko, Japan) and GlaxoSmithKline (Philadelphia, PA). The other authors did not report any potential conflicts of interest.

CLINICAL TRIAL REGISTRATION: ClinicalTrials.gov, www.clinicaltrials.gov, NCT00065845.

LEVEL OF EVIDENCE: II 
relationship with a patient's assessment of overall improvement, while anatomic success alone does not.

The lack of standardized definitions for surgical success following pelvic organ prolapse (POP) surgery has resulted in highly variable estimates of success. ${ }^{1,2}$ As an initial step forward, the 2001 National Institutes of Health (NIH) Workshop on Standardization of Terminology for Researchers in Pelvic Floor Disorders recommended arbitrary definitions for treatment success. ${ }^{3,4}$ The definition of "optimal anatomic outcome," designated as "cure," requires perfect anatomic support (Pelvic Organ Prolapse Quantification System [POP-Q] stage 0) and "satisfactory anatomic outcome" requires support higher than $1 \mathrm{~cm}$ proximal to the hymen. However, data now suggest that $75 \%$ of women presenting for annual gynecologic exams without symptoms of POP would not meet the definition of "optimal anatomic outcome" and almost $40 \%$ would not meet the definition of "satisfactory anatomic outcome." 5 Since the NIH Standardization Workshop, considerable variability in the definition of treatment success after surgery for prolapse still persists, making it difficult to compare study results. ${ }^{1,6}-9$

The objective of this study was to describe different definitions of surgical success after surgery for stages 2 to 4 POP in women undergoing abdominal sacrocolpopexy with or without Burch colposuspension enrolled in the Colpopexy and Urinary Reduction Efforts (CARE) trial. ${ }^{10,}$

11 Specifically, our objectives were to 1) describe how using different definitions affect estimates of treatment success; 2) evaluate how using different definitions affect comparisons of anatomic success between those who received a Burch colposuspension and those who did not; 3) compare different definitions of surgical success by examining their relationship to patients' subjective assessments of improvement, treatment success, and improvements in health-related quality of life; and 4) assess which definitions of treatment success result in the least amount of missing data 2 years after surgery. Information from this study should aid in planning future trials of prolapse surgery including sample size estimates and hopefully will ultimately contribute toward the development of a consensus definition of success after surgical treatment of prolapse.

\section{MATERIALS AND METHODS}

We analyzed data from 322 women who completed 2-year follow-up in the CARE trial, a randomized trial designed to evaluate whether a standardized modified Burch colposuspension, when added to abdominal sacrocolpopexy to treat POP, improves urinary stress continence in women without preoperative symptoms of stress urinary incontinence. ${ }^{10,11}$ Each clinical site and the data coordinating center received institutional review board approval for this trial, and all participants provided written informed consent.

The methods and primary outcomes of this trial have been previously reported. ${ }^{10,11}$ Colpopexy and Urinary Reduction Efforts trial participants were assessed before surgery and at 2-year follow-up for pelvic organ support using the POP-Q. ${ }^{4}$ In addition, pelvic symptoms and severity (Pelvic Floor Distress Inventory) along with condition-specific life impact (Pelvic Floor Impact Questionnaire) were measured via validated instruments. ${ }^{12}$ Two years after surgery, women rated the overall success of their treatment ("In your opinion, has the treatment of your pelvic floor condition been ?") on a 4-point scale (very successful, moderately successful, somewhat successful, not at all successful) and similarly a global assessment of improvement ("Compared with how you were doing before your recent pelvic floor operation, would you say that now you are ?") on a 5-point scale (much better, a little better, about the same, a little worse, much worse).

We created 18 different definitions of surgical success by using POP-Q assessments, responses to Pelvic Floor Distress Inventory questions regarding vaginal bulging, data on re-treatment 
(surgery or pessary), and participant's subjective ratings of overall treatment success and global improvement as listed in Table 1. The proposed definitions of treatment success consist of those recommended in the NIH Standardization Workshop and several used by clinical trials or prospective cohorts evaluating treatment success after prolapse surgery including one from an ongoing clinical trial being conducted by the Pelvic Floor Disorders Network (the OPTIMAL Trial). $1,2,7-9,13$

Definitions assessing anatomic outcomes used POP-Q measurements at 2-year follow-up to define treatment success or failure. To evaluate definitions that used the Baden-Walker system, 14 POP-Q measurements were used to approximate Baden-Walker grades 0-3. For instance, treatment success defined as Baden-Walker grade 0,1 , or 2 translates into "no descent of the vaginal walls beyond the hymen" or POP-Q measurements $\mathrm{Ba}, \mathrm{Bp}$, and $\mathrm{C}$ less than or equal to 0 . Treatment success defined as Baden-Walker grade 0 or 1 corresponds to "no vaginal descent beyond the half-way point of the vagina." For the apex, this translates into a POP-Q measurement of point $C$ that does not descend more than one-half the total vaginal length (Point $\mathrm{C}$ less than or equal to $1 / 2$ total vaginal length). For the anterior and posterior vaginal segments, there is no obvious direct translation to POP-Q measurements, so we assigned the criteria for success for the anterior/posterior segments as $\mathrm{Bp}$ and $\mathrm{Ba}$ less than -1 for this definition of treatment success to best approximate the Baden-Walker system.

Subjective cure was defined as the absence of vaginal bulge symptoms as indicated by a negative response at 2-year follow-up to the questions "Do you usually have a sensation of bulging or protrusion from the vaginal area?" and "Do you usually have a bulge or something falling out that you can see or feel in the vaginal area?" from the Pelvic Floor Distress Inventory. 12 We chose this definition of subjective cure as the patient's ability to see or feel a vaginal bulge as the symptom most consistently related to the presence or absence of POP. ${ }^{15}$

Re-treatment for POP was defined as any reoperation or use of pessary for recurrent POP over the 2-year follow-up period. Definitions of treatment success compositing anatomic outcomes, subjective cure, and/or re-treatment were also considered.

For each of 18 dichotomous definitions of treatment success, the proportion of women who had a successful outcome was reported for all analyzed CARE women. This cure rate was further compared between the women who received a Burch colposuspension and those who did not for each proposed definition by using $\chi^{2}$ or Fisher exact test. Additionally, the proportion of women with missing data that precluded a determination of success or failure for each definition was also determined. To evaluate the clinical relevance of each definition from a patient's perspective, we examined the relationship of subjective assessment of treatment success and the global impression of improvement to different definitions of surgical treatment success by comparing the assessments between those who met the criteria of treatment success to those who did not. Likewise, the comparisons between surgical success and failure groups were performed on the scores of the prolapse scales of the Pelvic Floor Distress Inventory and Pelvic Floor Impact Questionnaire (Pelvic Organ Prolapse Distress Inventory and Pelvic Organ Prolapse Impact Questionnaire). A clinically relevant definition of surgical success should demonstrate significantly better global impression of improvement, lower symptom bother (lower Pelvic Floor Distress Inventory scores), and higher health-related quality of life (lower Pelvic Floor Impact Questionnaire scores) in treatment successes than in failures. Mean and standard deviation were reported for the ordinal outcomes of subjective assessments and Pelvic Organ Prolapse Distress Inventory and Pelvic Organ Prolapse Impact Questionnaire scores, and the Wilcoxon rank sum test was used to identify any significant difference in outcomes between success and failure. All reported $P$ values were based on the two-sided statistical tests and intended to be interpreted from a hypothesis-generating framework. The analyses were performed in SAS 9.1.3 for Windows (SAS Inc., Cary, NC). 


\section{RESULTS}

Three hundred twenty-two women were enrolled in CARE with 157 women assigned to Burch colposuspension and 165 to the control group. All enrolled participants completed at least a portion of the 2-year follow-up that allowed assessment of at least one definition of treatment success considered in this analysis; 53 women $(16.5 \%)$ completed only questionnaire data at year 2 , and 24 women (7.4\%) only provided data on re-treatment. At baseline, $13.7 \%$ had stage 2 POP, $67.4 \%$ at stage 3, and $18.9 \%$ at stage 4 . One hundred twenty-six women $(39.1 \%)$ had a history of surgery for POP before enrollment in CARE. Further demographic information can be found elsewhere. ${ }^{10}$

Treatment success varied widely depending on definition used (19.2-97.2\%) (Table 1). Definitions of success requiring anatomic support proximal to the hymen had the lowest treatment success (19.2-57.6\%). In contrast, 94.3\% achieved surgical success when it was defined as the absence of prolapse beyond the hymen. Subjective cure (absence of bulge symptoms) occurred in $92.1 \%$ while absence of re-treatment occurred in $97.2 \%$ of participants. Surgery was considered "very successful" by $71.4 \%$ of women, and $85.2 \%$ of women considered themselves "much better" than before surgery on the global impression of improvement. The distributions of maximum descent of the anterior, posterior, and apical vaginal segments are displayed in Figure 1.

Missing data were more frequent for definitions requiring POP-Q values (24-25\%) than for those requiring data from patient interview alone $(0-10 \%)$. No significant difference in successful treatment of POP was found in those women who received a Burch compared with those who did not for each of the 18 definitions considered (data not shown).

Figure 2 demonstrates the relative size and overlap of the failures between two anatomic definitions of success (NIH satisfactory outcome, "no prolapse beyond the hymen"), subjective cure, and the proportion requiring no re-treatment 2 years after surgery in the 240 women who could provide data for all of these definitions. In this group, $45 \%$ failed to meet the NIH satisfactory anatomic criteria while $9 \%$ had prolapse beyond the hymen, $10 \%$ had symptoms of vaginal bulging, and $4 \%$ required re-treatment for prolapse recurrence. Vaginal bulging symptoms were noted in $18.5 \%$ (20 of 108) of those who did not meet the NIH satisfactory anatomic criteria and 64\% (14 of 22) of those with prolapse beyond the hymen. Seventeen percent ( 4 of 24) of participants with vaginal bulge symptoms had stage 0 or 1 pelvic organ support.

A comparison of the subjective assessment of treatment success and the global impression of improvement between treatment successes and failures for each definition considered is shown in Table 2. For each definition that was based solely on anatomic outcomes, we did not detect a significant difference in the patients' subjective assessment of treatment success or overall improvement between those who were considered a surgical success and those considered failures ( $P=.09-.99)$. In contrast, subjective cure (absence of vaginal bulging symptoms) was associated with significant improvements in the patient's assessment of both treatment success and overall improvement, more so than any other definition considered $(P<.001$ and $<.001$, respectively) When the absence of bulge symptoms was combined with the NIH satisfactory anatomic outcome criteria (POP-Q stage 0 and 1 ) to define treatment success, no significant relationship was noted between the patients' assessments of treatment success and overall improvement. In contrast, when the absence of bulge symptoms was combined with less strict anatomic criteria, significant relationships were noted ( $P \leq .05$ for each).

A comparison of the Pelvic Organ Prolapse Distress Inventory and Pelvic Organ Prolapse Impact Questionnaire scores between those who met the criteria for treatment success and those who did not for each definition considered is shown in Table 3 . The greatest difference in 
symptom burden and health-related quality of life as measured by the Pelvic Organ Prolapse Distress Inventory and Pelvic Organ Prolapse Impact Questionnaire scores between treatment successes and failures was noted when success was defined as subjective cure ( -55 and -29 points, respectively, $P<.001)$. Each of the seven definitions that combined anatomic criteria with absence of vaginal bulge symptoms to define success demonstrated significantly less symptom burden in treatment successes than in failures compared with only two of the seven definitions that used anatomic criteria alone. Health-related quality of life as measured by Pelvic Organ Prolapse Impact Questionnaire was significantly better in treatment successes than failures in four of seven definitions that combined subjective cure with anatomic criteria but in none of the definitions that used anatomic criteria alone. When the NIH optimal anatomic outcome was used, health-related quality of life was significantly worse in treatment successes than in failures (difference in Pelvic Organ Prolapse Impact Questionnaire: $+10.5, P=.02$ ).

\section{DISCUSSION}

In this study, we demonstrated that cure rates 2 years after abdominal sacrocolpopexy vary dramatically depending on the definition of treatment success used. Almost three-fourths of participants considered their surgery "very successful," re-treatment was uncommon (2.8\%), and less than $10 \%$ of participants complained of symptomatic vaginal bulging postoperatively, yet cure rates using strict anatomic criteria such as those found in the NIH Workshop definitions of optimal and satisfactory anatomic outcome were only $19 \%$ and 57\%, respectively. Cure rates using definitions with less stringent anatomic criteria for all segments or that considered only support of the vaginal apex were appreciably higher (90.6-95.5\%) and more consistent with subjective cure rates. This is consistent with a recent systematic review of sacrocolpopexy in which the success rates ranged from 58\% to 100\%; studies with the lowest success rates defined success using more stringent anatomic support while those with the highest success rates generally used subjective outcomes such as satisfaction and resolution of POP symptoms. $7,20-22$

The NIH Workshop recommended that, until more data became available, only the complete absence of prolapse should be considered "normal"; hence their recommendation for the optimal anatomic outcome. ${ }^{3}$ More information is now available, about the relationship between pelvic organ support and symptoms. Most symptoms often attributing to POP have at best weak-to-moderate correlations with worsening pelvic organ support; however, the one symptom that is almost consistently acknowledged by patients with advanced POP is the presence of a vaginal bulge that can be seen or felt. ${ }^{15,23-26}$ Additionally, the hymen seems to be an important "cut-off point" as women with prolapse beyond the hymen have more pelvic floor symptoms and are more likely to report a vaginal bulge than women with prolapse at or above the hymen. $5,15,23-26$

In addition, we now know that the majority of women presenting for routine care or evaluated in population-based research demonstrate some loss of vaginal or uterine support on pelvic examination and 3-6\% have descent of the prolapse beyond the hymen. ${ }^{5,27}$ Given this, the NIH Workshop definitions of optimal (POP-Q stage 0 ) and satisfactory anatomic outcome (POP-Q stage 1) no longer seem appropriate because a substantial proportion of women in the general population without symptoms of POP would not meet these criteria.

One of the most important findings in our study is that definitions that included the absence of vaginal bulge symptoms had the strongest relationships with the patients' assessment of overall improvement and treatment success as well as improvements in symptom bother and healthrelated quality of life. Few of the definitions using anatomic criteria alone to define treatment success demonstrated any such relationships, and when they did they were relatively weak. Thus, as a general rule, definitions of treatment success that require patients be free of vaginal 
bulging symptoms postoperatively are more clinically relevant and meaningful to the patient than those that include only anatomic criteria to define success. This, in our opinion, is a strong argument for including the absence of vaginal bulge symptoms in any consensus definition of success after treatment of POP. However, paradoxically, $17 \%$ of participants in our population who reported vaginal bulge symptoms demonstrated good anatomic support (stages 0 or 1). Further research is necessary to understand the origin and clinical significance of these symptoms.

The clinical relevance of stage 2 prolapse that is asymptomatic is unclear. If a high proportion of women with stage 2 POP postoperatively progress over time and require further surgery and if the same is not true for women with stages 0 or 1 prolapse, then early anatomic differences gain greater clinical significance; if the rate of symptomatic progression in this group is low then the differences seen are less clinically meaningful. Surgical trials with long-term followup are critical to determine the clinical relevance of this group.

In conclusion, an ideal outcome measure should be valid, reliable, responsive, easily assessed, easily interpreted, and clinically relevant. Good outcome measures are essential to compare one treatment with another, to compare our own results with those of another and, perhaps most importantly, to counsel our patients effectively, realistically, and honestly using outcomes that are relevant to the individual patient. ${ }^{16}$ The results of this study along with the emerging literature on the distribution of pelvic support loss in the general population and data on the relationship between pelvic organ support loss and symptom development lead us to make the following recommendations: 1) any definition of success after POP surgery should include the absence of bulge symptoms in addition to anatomic criteria and the absence of re-treatment and 2) using the hymen as a threshold for anatomic success seems a reasonable and defensible approach. However, this approach should be revisited when we have an understanding, based on a larger body of long-term data, of the significance, or lack thereof, of asymptomatic stage 2 POP 1-2 years after surgery. Further studies are also needed to understand the significance of recurrent apical compared to anterior or posterior vaginal support defects. An anterior vaginal wall supported $2 \mathrm{~cm}$ above the hymen has dropped $1 \mathrm{~cm}$ from "perfect," while an apex supported $2 \mathrm{~cm}$ above the hymen has dropped $5 \mathrm{~cm}$ or more, yet both are currently categorized as stage 1 POP. As more data become available about the issues discussed above, surgeons will be able to improve on definitions of success to create a valid and reproducible surgical outcome measure. In the meantime, when counseling women about surgical success rates for a given procedure, surgeons should describe "success" using a patient perspective to ensure a mutually agreeable definition of an acceptable outcome before the intervention. Perioperative goal setting and achievement may augment this patient-surgeon communication.

\section{Acknowledgments}

Supported by grants from the Eunice Kennedy Shriver National Institute of Child Health and Human Development; National Institute of Diabetes, Digestive and Kidney Diseases; and the National Institutes of Health Office of Research on Women's Health (U01 HD41249, U10 HD41250, U10 HD41261, U10 HD41267, U10 HD54136, U10 HD54214, U10 HD54215, and U10 HD54241).

\section{References}

1. Brubaker, L.; Bump, RC.; Fynes, M.; Jacquetin, B.; Karram, M.; Kreder, K., et al. In: Abrams, P.; Cordozo, L.; Koury, S.; Wein, A., editors. Surgery for pelvic organ prolapse; 3rd international consultation on incontinence; Paris: Health Publication Ltd; 2005. p. 1371-1401.

2. Maher C, Baessler K, Glazener CM, Adams EJ, Hagen S. Surgical management of pelvic organ prolapse in women. Cochrane Database Syst Rev 2004:CD004014. [PubMed: 15495076] 
3. Weber AM, Abrams P, Brubaker L, Cundiff G, Davis G, Dmochowski RR, et al. The standardization of terminology for researchers in female pelvic floor disorders. Int Urogynecol J Pelvic Floor Dysfunct 2001;12:178-86. [PubMed: 11451006]

4. Bump RC, Mattiasson A, Bo K, Brubaker LP, DeLancey JO, Klarskov P, et al. The standardization of terminology of female pelvic organ prolapse and pelvic floor dysfunction. Am J Obstet Gynecol 1996;175:10-7. [PubMed: 8694033]

5. Swift S, Woodman P, O’Boyle A, Kahn M, Valley M, Bland D, et al. Pelvic Organ Support Study (POSST): the distribution, clinical definition, and epidemiologic condition of pelvic organ support defects. Am J Obstet Gynecol 2005;192:795-806. [PubMed: 15746674]

6. Weber AM, Walters MD, Piedmonte MR, Ballard LA. Anterior colporrhaphy: a randomized trial of three surgical techniques. Am J Obstet Gynecol 2001;185:1299-304. [PubMed: 11744900]

7. Maher CF, Qatawneh AM, Dwyer PL, Carey MP, Cornish A, Schluter PJ. Abdominal sacral colpopexy or vaginal sacrospinous colpopexy for vaginal vault prolapse: a prospective randomized study. Am J Obstet Gynecol 2004;190:20-6. [PubMed: 14749629]

8. Paraiso MF, Ballard LA, Walters MD, Lee JC, Mitchinson AR. Pelvic support defects and visceral and sexual function in women treated with sacrospinous ligament suspension and pelvic reconstruction. Am J Obstet Gynecol 1996;175:1423-30. [PubMed: 8987920]

9. Barber MD, Visco AG, Weidner AC, Amundsen CL, Bump RC. Bilateral uterosacral ligament vaginal vault suspension with site-specific endopelvic fascia defect repair for treatment of pelvic organ prolapse. Am J Obstet Gynecol 2000;183:1402-10. [PubMed: 11120503]

10. Brubaker L, Cundiff GW, Fine P, Nygaard I, Richter HE, Visco AG, et al. Abdominal sacrocolpopexy with Burch colposuspension to reduce urinary stress incontinence. N Engl J Med 2006;354:155766. [PubMed: 16611949]

11. Brubaker L, Cundiff G, Fine P, Nygaard I, Richter H, Visco A, et al. A randomized trial of colpopexy and urinary reduction efforts (CARE): design and methods. Control Clin Trials 2003;24:629-42. [PubMed: 14500059]

12. Barber MD, Kuchibhatla MN, Pieper CF, Bump RC. Psychometric evaluation of 2 comprehensive condition-specific quality of life instruments for women with pelvic floor disorders. Am J Obstet Gynecol 2001;185:1388-95. [PubMed: 11744914]

13. Barber MD, Brubaker L, Menefee S, Norton P, Borello-France D, Varner E, et al. Operations and pelvic muscle training in the management of apical support loss (OPTIMAL) trial: design and methods. Contemp Clin Trials 2009;30:178-89. [PubMed: 19130903]

14. Baden WF, Walker TA. Genesis of the vaginal profile: a correlated classification of vaginal relaxation. Clin Obstet Gynecol 1972;15:1048-54. [PubMed: 4649139]

15. Barber MD. Symptoms and outcome measures of pelvic organ prolapse. Clin Obstet Gynecol 2005;48:648-61. [PubMed: 16012232]

16. Hilton P. Trials of surgery for stress incontinence-thoughts on the "Humpty Dumpty principle. BJOG 2002;109:1081-8. [PubMed: 12387459]

17. Barber MD, Walters MD, Bump RC. Short forms of two condition-specific quality-of-life questionnaires for women with pelvic floor disorders (PFDI-20 and PFIQ-7). Am J Obstet Gynecol 2005;193:103-13. [PubMed: 16021067]

18. Digesu GA, Khullar V, Cardozo L, Robinson D, Salvatore S. P-QOL: a validated questionnaire to assess the symptoms and quality of life of women with urogenital prolapse. Int Urogynecol J Pelvic Floor Dysfunct 2005;16:176-81. [PubMed: 15875234]

19. Price N, Jackson SR, Avery K, Brookes ST, Abrams P. Development and psychometric evaluation of the ICIQ Vaginal Symptoms Questionnaire: the ICIQ-VS. BJOG 2006;113:700-12. [PubMed: 16709214]

20. Nygaard IE, McCreery R, Brubaker L, Connolly A, Cundiff G, Weber AM, et al. Abdominal sacrocolpopexy: a comprehensive review. Obstet Gynecol 2004;104:805-23. [PubMed: 15458906]

21. Benson JT, Lucente V, McClellan E. Vaginal versus abdominal reconstructive surgery for the treatment of pelvic support defects: a prospective randomized study with long-term outcome evaluation. Am J Obstet Gynecol 1996;175:1418-21. discussion 1421-2. [PubMed: 8987919]

22. Lo TS, Wang AC. Abdominal colposacropexy and sacrospinous ligament suspension for severe uterovaginal prolapse: a comparison. J Gynecol Surg 1998;14:59-64. 
23. Swift SE, Tate SB, Nicholas J. Correlation of symptoms with degree of pelvic organ support in a general population of women: what is pelvic organ prolapse? Am J Obstet Gynecol 2003;189:3727. [PubMed: 14520198]

24. Bradley CS, Nygaard IE. Vaginal wall descensus and pelvic floor symptoms in older women. Obstet Gynecol 2005;106:759-66. [PubMed: 16199633]

25. Ellerkmann RM, Cundiff GW, Melick CF, Nihira MA, Leffler K, Bent AE. Correlation of symptoms with location and severity of pelvic organ prolapse. Am J Obstet Gynecol 2001;185:1332-7. [PubMed: 11744905]

26. Tan JS, Lukacz ES, Menefee SA, Powell CR, Nager CW. Predictive value of prolapse symptoms: a large database study. Int Urogynecol J Pelvic Floor Dysfunct 2005;16:203-9. [PubMed: 15875236]

27. Samuelsson EC, Victor FT, Tibblin G, Svardsudd KF. Signs of genital prolapse in a Swedish population of women 20 to 59 years of age and possible related factors. Am J Obstet Gynecol 1999;180:299-305. [PubMed: 9988790]

28. Paraiso MF, Barber MD, Muir TW, Walters MD. Rectocele repair: a randomized trial of three surgical techniques including graft augmentation. Am J Obstet Gynecol 2006;195:1762-71. [PubMed: 17132479]

29. Hiltunen R, Nieminen K, Takala T, Heiskanen E, Merikari M, Niemi K, et al. Low-weight polypropylene mesh for anterior vaginal wall prolapse: a randomized controlled trial. Obstet Gynecol 2007;110:455-62. [PubMed: 17666627] 

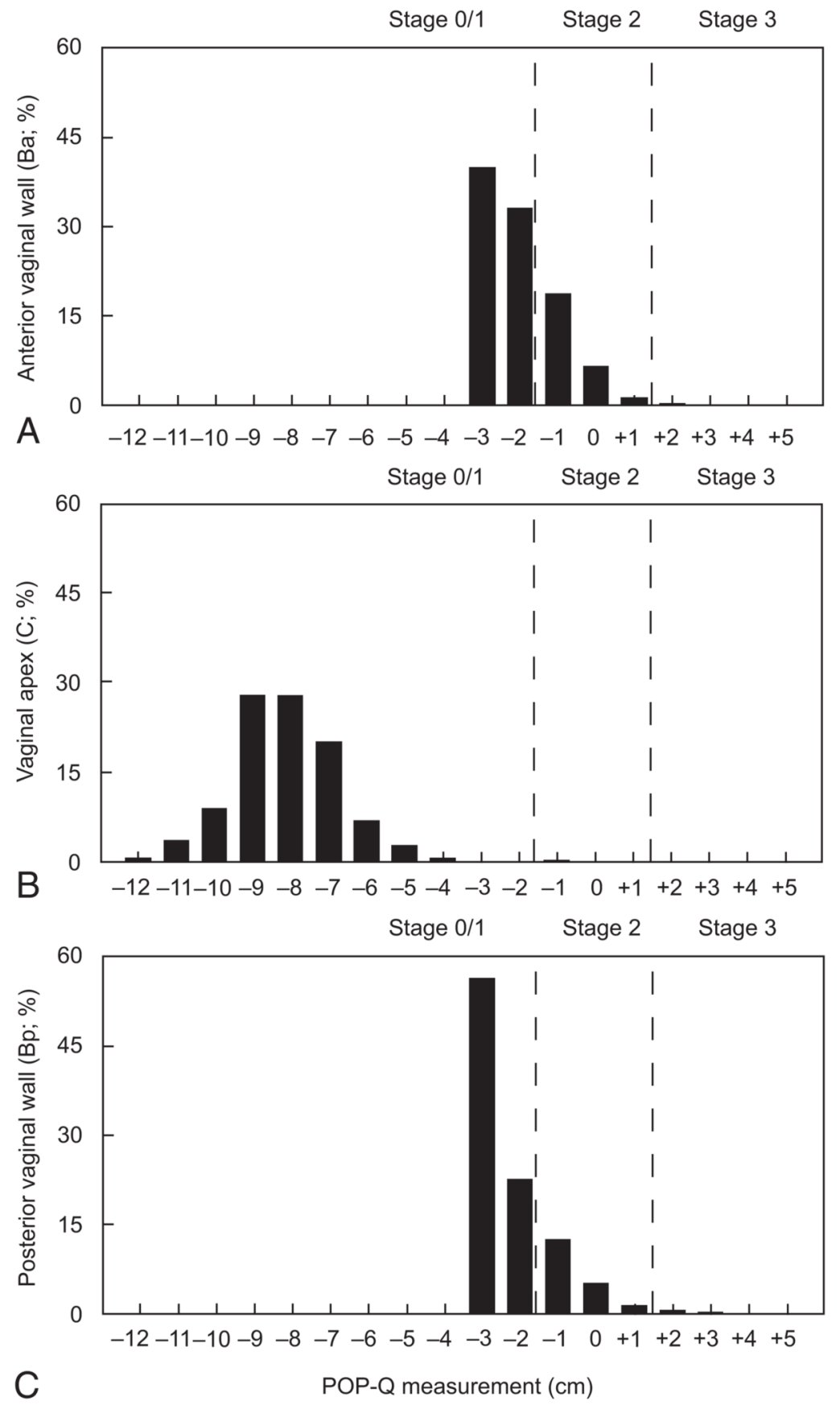

Fig. 1.

Distribution of the maximum descent of the anterior (A), apical (B), and posterior $(\mathbf{C})$ vaginal segments 2 years after surgery. Pelvic organ prolapse quantification (POP-Q) measurements are made in $1-\mathrm{cm}$ increments with descent to the hymen equal to 0 , with negative numbers indicating support proximal to the hymen, and positive numbers indicating prolapse beyond the hymen. Point $\mathrm{Ba}$, maximum descent of anterior vaginal wall; point $\mathrm{C}$, maximum descent of the cervix or vaginal cuff; point $\mathrm{Bp}$, maximum posterior vaginal wall. Perfect anterior and posterior vaginal wall support corresponds to a value of -3 for $\mathrm{Ba}$ and $\mathrm{Bp}$, respectively.

Corresponding POP-Q stages are listed across the top.

Barber. Defining Success After Prolapse Surgery. Obstet Gynecol 2009. 


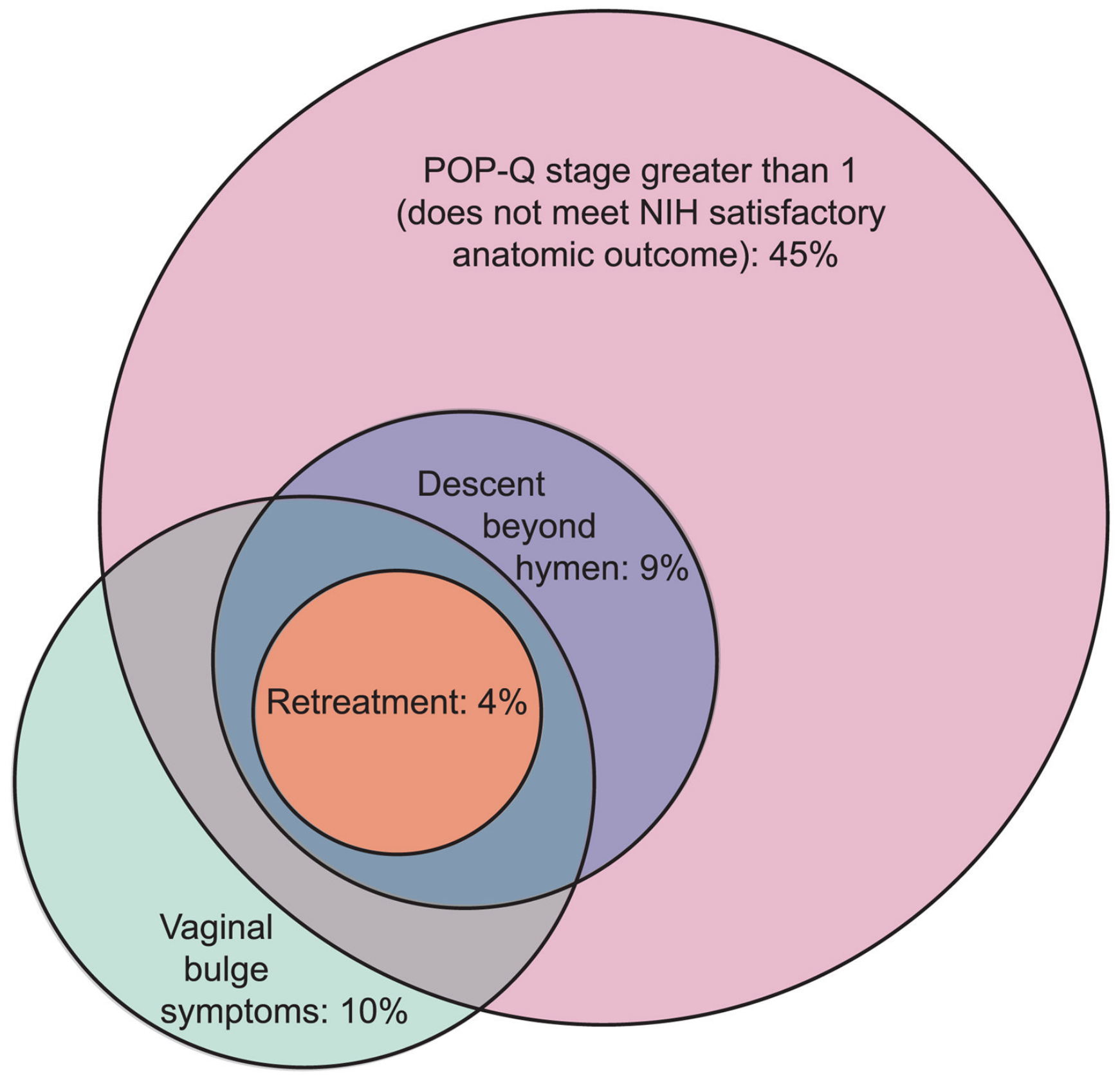

Fig. 2.

Venn diagram of failures rates (in parentheses) using four definitions of success: two anatomic definitions (National Institutes of Health [NIH] "satisfactory" anatomic outcome [pelvic organ prolapse quantification (POP-Q) stage 0 or 1] and "no descent beyond the hymen"), subjective cure, and no re-treatment. Universe union of $\mathrm{N}=240$ represents the participants who have data on all four definitions of success in the diagram. In the Venn diagram, we assume any retreatment over the 2-year follow-up also fails by every other definition as well. Barber. Defining Success After Prolapse Surgery. Obstet Gynecol 2009. 


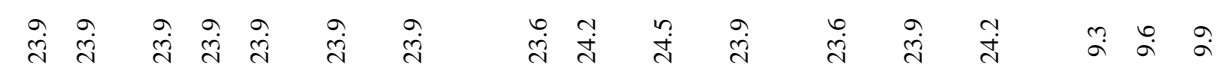

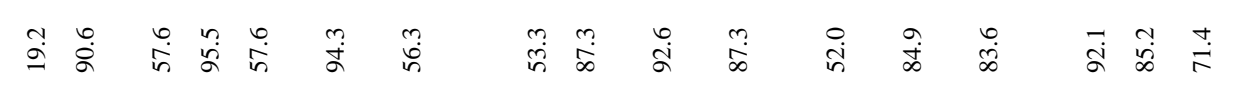


Barber et al.

Page 12

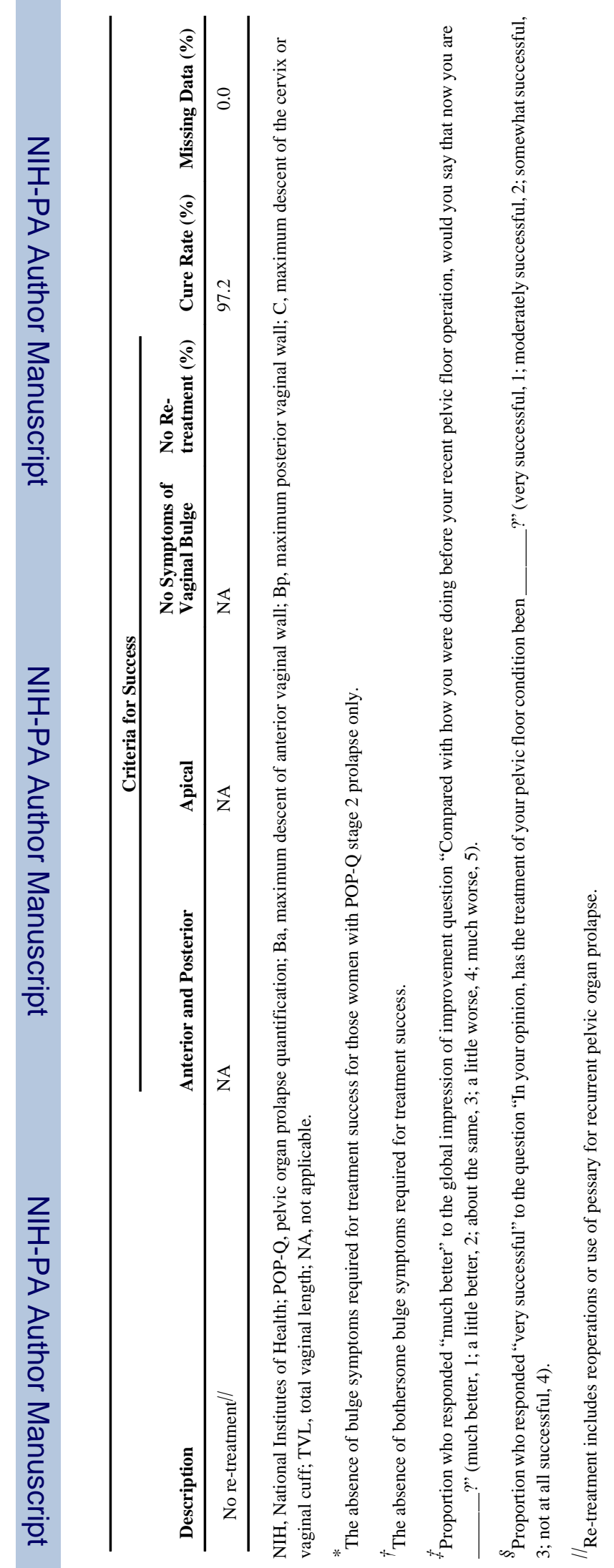

Obstet Gynecol. Author manuscript; available in PMC 2010 July 15. 


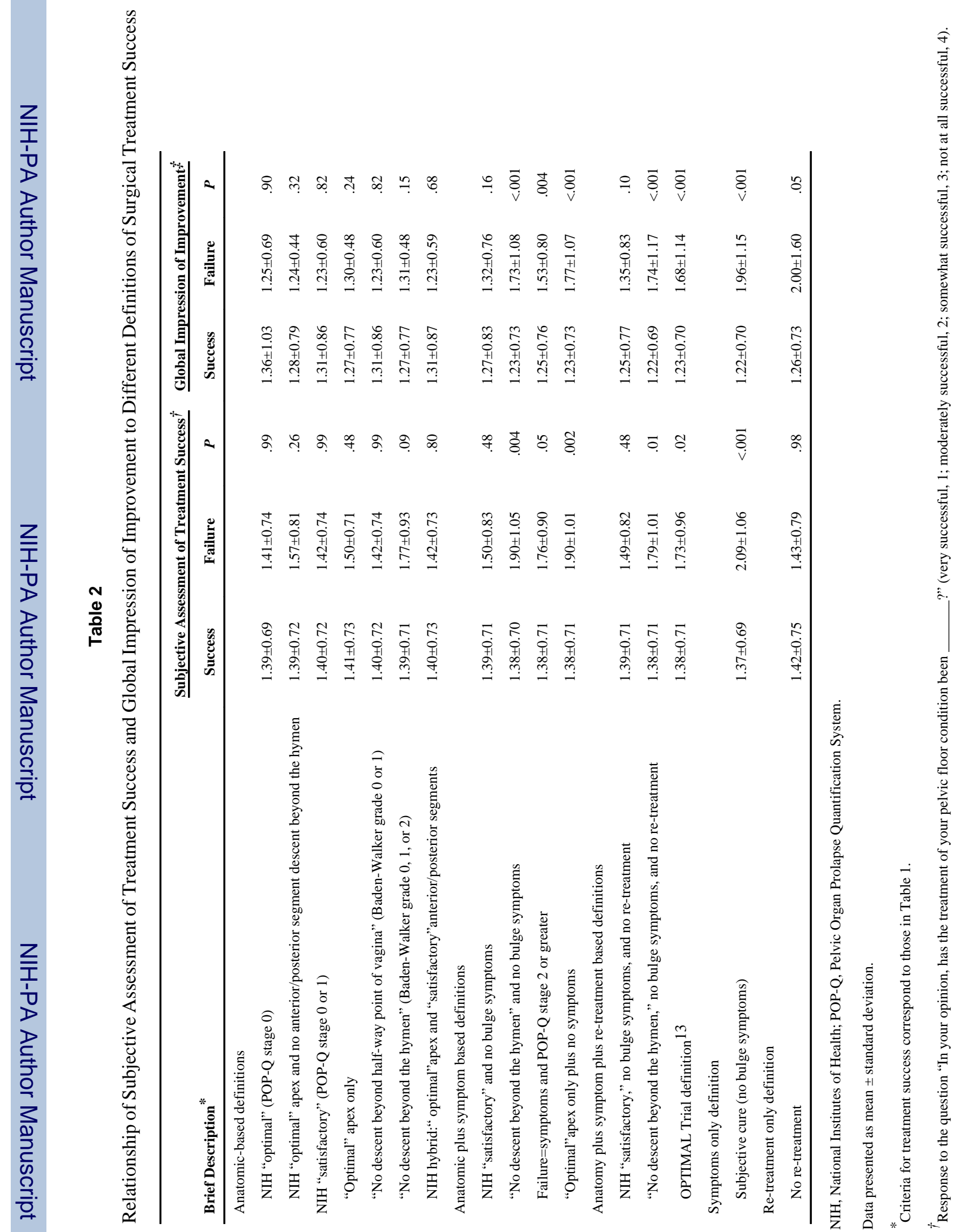




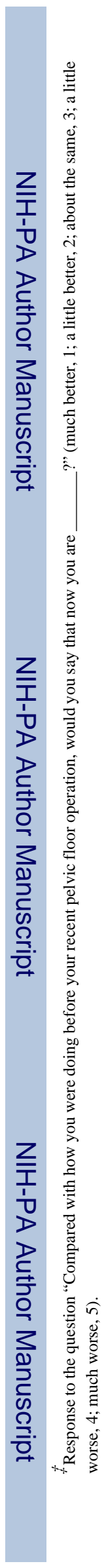

Barber et al.

Page 14

Obstet Gynecol. Author manuscript; available in PMC 2010 July 15. 


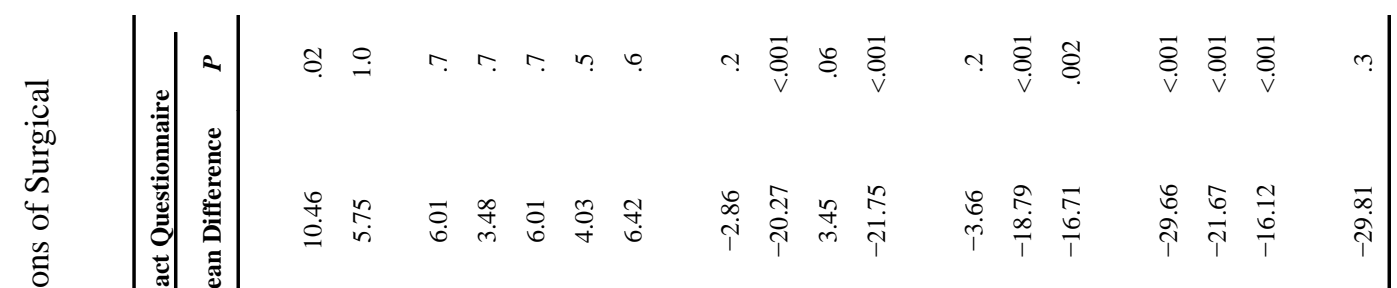

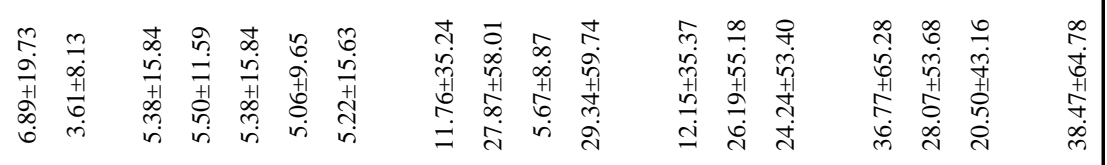

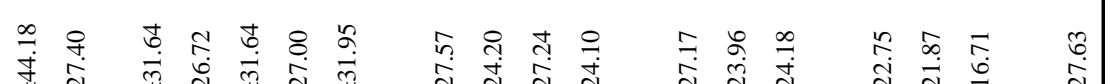

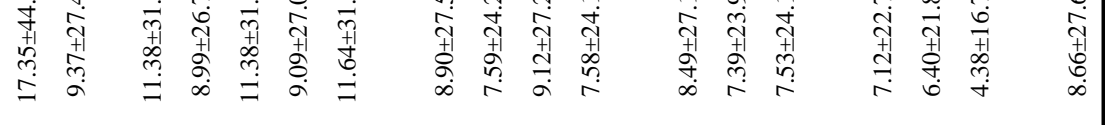

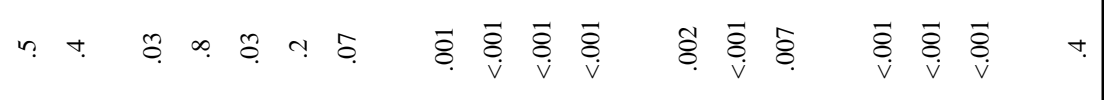

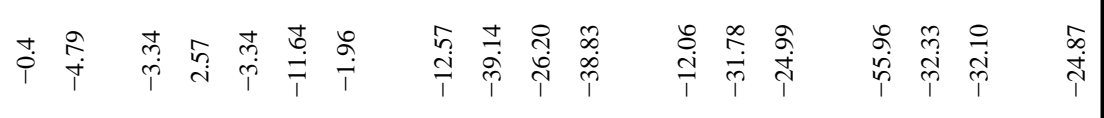

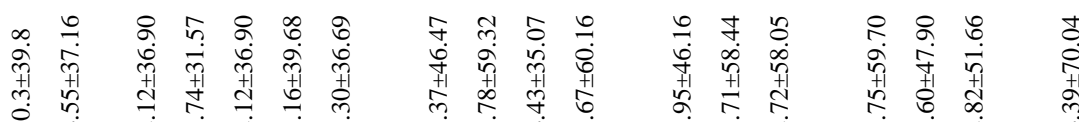

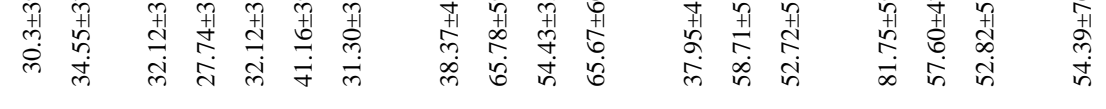

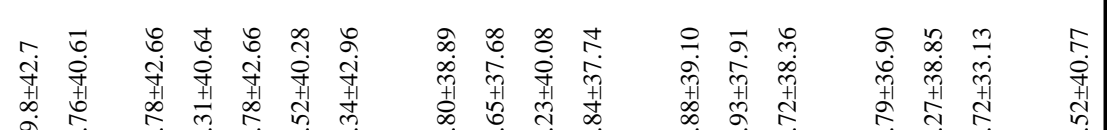

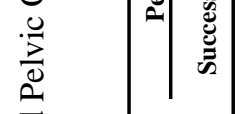

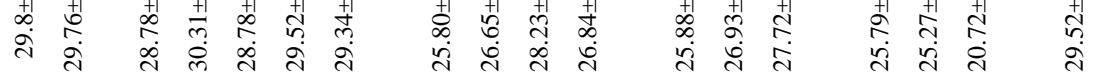
$\overline{7}$

है

:
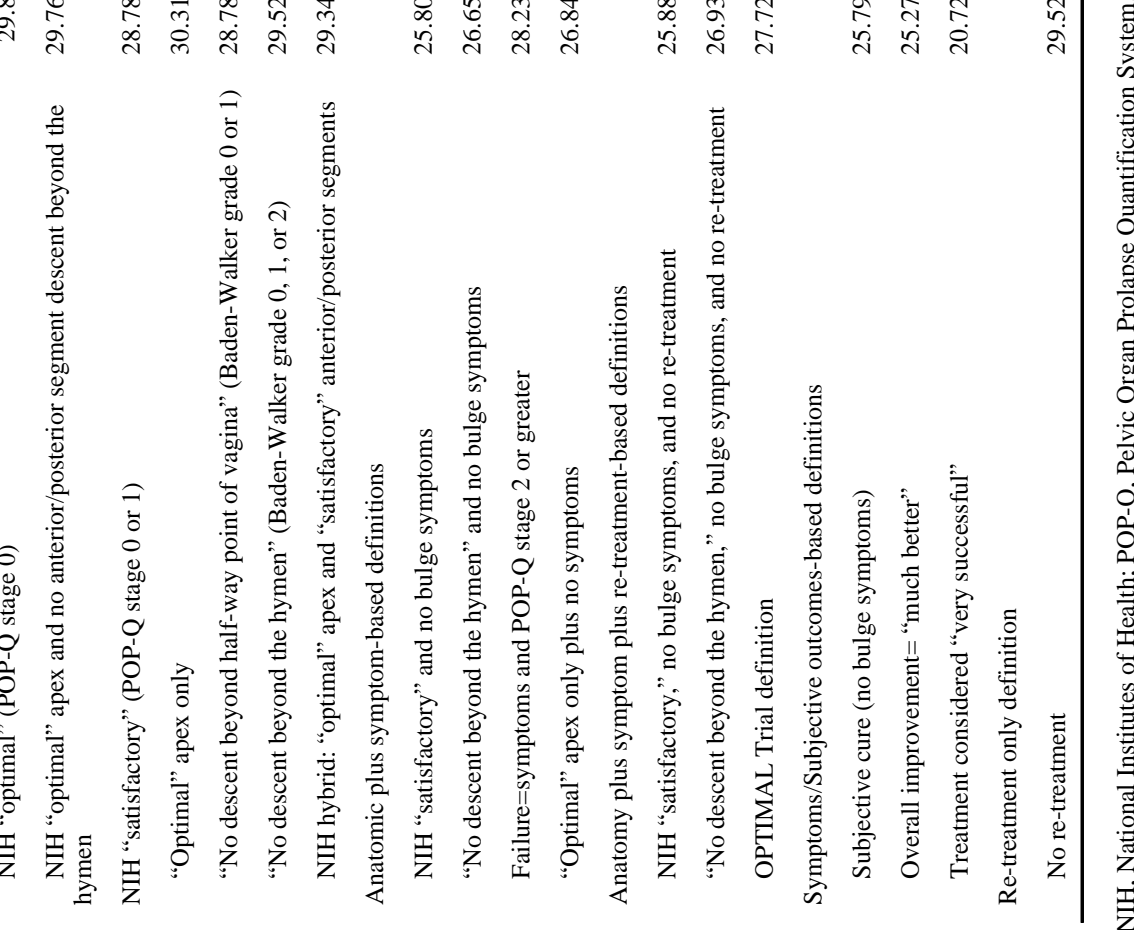

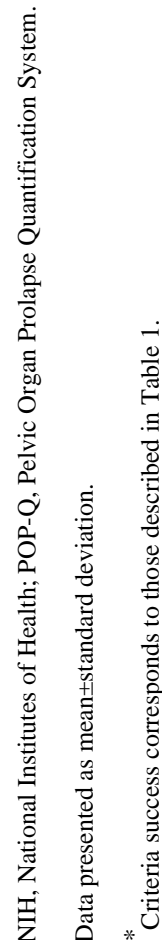

